\title{
Multi-Level Acceleration for Sub-Iterations in Partitioned Fluid-Structure Interaction
}

\author{
A.H. van Zuijlen and H. Bijl \\ Delft University of Technology, Kluyverweg 1, 2629HS, Delft, The Netherlands
}

\begin{abstract}
Computational fluid-structure interaction is most commonly performed using a partitioned approach. For strongly coupled problems sub-iterations are required, increasing computational time as flow and structure have to be resolved multiple times every time step. Many sub-iteration techniques exist that improve robustness and convergence, although still a flow and structure have to be solved a number of times every time step.

In this paper we apply a multi-level acceleration technique, which is based on the presumed existing multi-grid solver for the flow domain, to a two-dimensional strongly coupled laminar and turbulent problem and investigate the combination of multi-level acceleration with the Aitken underrelaxation technique. It is found that the value for the underrelaxation parameter is not significantly different when performing sub-iterations purely on the coarse level or purely on the fine level. Therefore coarse and fine level sub-iterations are used alternatingly, where it is found that performing 3 or 4 coarse level sub-iterations followed by 1 fine level sub-iteration resulted in the highest gain in efficiency. Although the total number of sub-iterations increases slightly by $25-30 \%$, the number of fine grid iterations can be decreased by as much as $65-70 \%$.
\end{abstract}

Keywords: Partitioned fluid-structure interaction, sub-iterations, multi-level acceleration

PACS: $02.60 .-\mathrm{Cb} ; 02.60 .-\mathrm{Pn} ; 47.11,-\mathrm{j}$

\section{INTRODUCTION}

In many engineering applications, fluid-structure interaction phenomena play a key role in the dynamic stability of a structure (e.g. aircraft, wind-turbines, suspension bridges, etc.). A fast and accurate computation of the dynamic interaction between flow and structure is, therefore, of the utmost importance. Computational fluid-structure interaction is most commonly performed using a partitioned approach, so that existing Computational Fluid Dynamics (CFD) and Computational Structural Dynamics (CSD) codes are coupled at the fluid-structure interface. Loosely coupled partitioned approaches suffer from numerical instability when applied to strongly coupled problems. In that case subiterations are required, increasing computational time as flow and structure have to be resolved multiple times every time step.

Basic sub-iteration techniques include block-Gauss-Seidel iterations, which may suffer instability or fixed underrelaxation methods, which are robust, but at the price of slower convergence. In literature several methods can be found for performing sub-iterations in an efficient and robust fashion. One of the most popular methods is the Aitken underrelaxation method [1], that tunes the underrelaxation parameter to obtain faster convergence.

Whichever method is chosen, a (small) number of sub-iterations still has to be performed. In previous research [2] we investigated the possibility of performing sub-iterations initially on a coarse level and showed that a basic coarse level block-Gauss-Seidel sub-iteration would have much the same convergence as sub-iterating on the fine level. In this paper we apply this model to a more challenging two-dimensional strongly coupled laminar [3] and turbulent problem and investigate the combination of multi-level acceleration with Aitken underrelaxation. We investigate the effectiveness of performing initial sub-iterations on a coarse level and alternating coarse and fine level sub-iterations.

\section{COUPLED PROBLEM}

First we introduce the coupled problem that we want to solve, without going into too much detail about the actual governing equations. Schematically, we can represent the discretized partitioned fluid-structure interaction problem that has to be solved at a certain time step as

$$
\begin{array}{r}
\mathbf{r}_{s}\left(\mathbf{w}_{s}, \mathbf{p}_{\Gamma_{s}}\right)-\mathbf{s}_{s}=\mathbf{0} \\
\mathbf{r}_{f}\left(\mathbf{w}_{f}, \mathbf{d}_{\Gamma_{f}}\right)-\mathbf{s}_{f}=\mathbf{0}
\end{array}
$$


wherein $\mathbf{r}$ is the residual function (discretized representation of the governing equations), $\mathbf{s}$ a constant source term within the time step that can depend on previous solutions or boundary conditions, $\mathbf{w}$ the state vector, $\mathbf{p}_{\Gamma}$ the discrete pressures in the boundary nodes and $\mathbf{d}_{\Gamma}$ the discrete displacements of the boundary nodes. The subscripts $s, f$ denote the discrete quantities belong to the structure and fluid domains, respectively. A CSD package is capable of finding a $\mathbf{w}_{S}$ such that (1) is satisfied for a given pressure load $\mathbf{p}_{\Gamma_{s}}$. A CFD package is able to find a $\mathbf{w}_{f}$ such that (2) is satisfied for a given boundary displacement $\mathbf{d}_{\Gamma_{f}}$.

The coupling between flow and structure takes place at the fluid-structure boundary. Let us assume that the coupling is performed by transferring displacements from the discrete structure boundary to the discrete flow boundary through an interpolation $\mathscr{I}_{f s}$

$$
\mathbf{d}_{\Gamma_{f}}=\mathscr{I}_{f s}\left(\mathbf{d}_{\Gamma_{s}}\right)
$$

and the displacements at the structure boundary follow directly from the structure state vector $\mathbf{d}_{\Gamma_{s}}=f\left(\mathbf{w}_{s}\right)$. The second part of the coupling is the transfer of pressure loads from the flow to the structure also by interpolation $\mathscr{I}_{f s}$

$$
\mathbf{p}_{\Gamma_{s}}=\mathscr{I}_{s f}\left(\mathbf{p}_{\Gamma_{f}}\right)
$$

and the pressure at the fluid boundary follows directly from the fluid state vector $\mathbf{p}_{\Gamma_{f}}=g\left(\mathbf{w}_{f}\right)$.

\section{Sub-iterations}

In partitioned fluid-structure interaction, obtaining the coupled solution described by (1) and (2) would require sub-iterating, e.g. when a sequential algorithm is used

$$
\begin{aligned}
\mathbf{r}_{s}\left(\mathbf{w}_{s}^{i}, \hat{\mathbf{p}}_{\Gamma_{s}}^{i}\right)-\mathbf{s}_{s} & =\mathbf{0} \\
\mathbf{r}_{f}\left(\mathbf{w}_{f}^{i}, \mathscr{I}_{f s}\left(g\left(\mathbf{w}_{s}^{i}\right)\right)\right)-\mathbf{s}_{f} & =\mathbf{0}
\end{aligned}
$$

wherein the superscript $i$ denotes the $i$-th sub-iteration and $\hat{\mathbf{p}}_{\Gamma_{s}}^{i}$ is the estimation of the fluid pressure acting on the structure for the $i$-th iteration. The simplest choice for the estimation is $\hat{\mathbf{p}}_{\Gamma_{s}}^{i}=\mathscr{I}_{s f}\left(g\left(\mathbf{w}_{f}^{i-1}\right)\right)$, which results in a blockGauss-Seidel type of iteration, but which is not guaranteed to be stable. To increase robustness underrelaxation can be applied, but generally at the expense of slower convergence rate. In this paper we focus on the widely applied Aitken method, which applies an adaptive underrelaxation to the estimation for the next time step

$$
\hat{\mathbf{p}}_{\Gamma_{s}}^{i+1}=\hat{\mathbf{p}}_{\Gamma_{s}}^{i}+\theta^{i+1}\left(\mathbf{p}_{\Gamma_{s}}^{i}-\hat{\mathbf{p}}_{\Gamma_{s}}^{i}\right)
$$

for which the underrelaxation parameter $\theta^{i+1}$ is obtained from

$$
\theta^{i+1}=\theta^{i}\left(1-\frac{\left(\Delta \mathbf{e}^{i}\right)^{T}\left(\mathbf{e}^{i}\right)}{\left(\Delta \mathbf{e}^{i}\right)^{T}\left(\Delta \mathbf{e}^{i}\right)}\right)
$$

with $\mathbf{e}^{i}=\mathbf{p}_{\Gamma_{s}}^{i}-\hat{\mathbf{p}}_{\Gamma_{s}}^{i}$ the error between the estimated and the resulting pressure after solving (5) and (6) for iteration $i$ and $\Delta \mathbf{e}^{i}=\mathbf{e}^{i}-\mathbf{e}^{i-1}$. For the first underrelaxation step a $\theta$ has to be chosen as $\mathbf{e}^{i-1}$ is not available yet. One can use last known value and at the very start of the computation any (sufficiently small) value can be taken.

\section{TWO-LEVEL ACCELERATION}

In order to reduce the computational time for the (initial) sub-iterations, the two-level acceleration scheme solves for a correction of the solution on a coarsened mesh. In this case we only consider coarsening the fluid mesh as this is usually the most computationally intensive solve for each sub-iteration. The algorithm consists of the following steps:

1. Solve the structure (5) using Aitken for the prediction of the pressure force (7),

2. Evaluate (but do not solve) the fluid equations with the fluid state still at the previous sub-iteration

$$
\mathbf{r}_{h, f}\left(\mathbf{w}_{h, f}^{i-1}, \mathscr{I}_{h, f S}\left(g\left(\mathbf{w}_{S}^{i}\right)\right)\right)-\mathbf{s}_{h, f}=\widetilde{\mathbf{r}}_{h, f}
$$


wherein the subscript $h$ denotes values on the fine fluid mesh. Subtraction of (9) from (6) yields

$$
\mathbf{r}_{h, f}\left(\mathbf{w}_{h, f}^{i}, \mathscr{I}_{h, f s}\left(g\left(\mathbf{w}_{s}^{i}\right)\right)\right)-\mathbf{r}_{h, f}\left(\mathbf{w}_{h, f}^{i-1}, \mathscr{I}_{h, f_{s}}\left(g\left(\mathbf{w}_{s}^{i}\right)\right)\right)=-\tilde{\mathbf{r}}_{h, f},
$$

which implicitly describes the correction $\mathbf{w}_{h, f}^{i}-\mathbf{w}_{h, f}^{i-1}$ for a given defect $\tilde{\mathbf{r}}_{h, f}$.

3. Solve an approximation of (10) on the coarse fluid mesh

$$
\mathbf{r}_{H, f}\left(\mathbf{w}_{H, f}^{i}, \mathscr{I}_{H, f s}\left(g\left(\mathbf{w}_{s}^{i}\right)\right)\right)-\mathbf{r}_{H, f}\left(\mathbf{w}_{H, f}^{i-1}, \mathscr{I}_{H, f s}\left(g\left(\mathbf{w}_{s}^{i}\right)\right)\right)=-\tilde{\mathbf{r}}_{H, f},
$$

wherein the subscript $H$ denotes values on the coarse fluid mesh and the coarse level fluid state and residual are obtained through a restriction operator $R: \mathbf{w}_{H, f}=R \mathbf{w}_{h, f}$ and $\tilde{\mathbf{r}}_{H, f}=R \tilde{\mathbf{r}}_{h, f}$. Iterating the flow solver to obtain $\mathbf{w}_{H, f}^{i}$ that satisfies (11) can be done relatively inexpensively compared to resolving (6) on the fine mesh. This results in a coarse grid estimation of the correction term $\Delta \mathbf{w}_{H, f}=\mathbf{w}_{H, f}^{i}-\mathbf{w}_{H, f}^{i-1}$.

4. Update the fluid state at the fine level by applying a correction by prolongation $(P)$ of $\Delta \mathbf{w}_{H, f}$ to the fine grid

$$
\mathbf{w}_{h, f}^{i}=\mathbf{w}_{h, f}^{i-1}+P \Delta \mathbf{w}_{H, f} .
$$

5. Continue the procedure starting at step 1 until sufficiently converged or the maximum number $n_{c}$ of coarse level iterations is performed.

6. Perform standard sub-iterations on the fine grid until sufficiently converged or the maximum number $n_{f}$ of fine level iterations is performed.

7. When not sufficiently converged continue at step 1.

The algorithm allows the subsequent use of coarse and fine grid sub-iterations and is denoted by $\mathrm{CGP}_{n_{c}} \mathrm{C}_{n_{f}}$, e.g. $\mathrm{CGP}_{1} \mathrm{C}_{1}$ means that 1 coarse level prediction step is performed followed by 1 fine level correction (smoothing) subiteration, whereas $\mathrm{CGP}_{\infty} \mathrm{C}_{\infty}$ (also denoted by CGP-FG) denotes that first all sub-iterations are performed on a coarse level until converged, thereafter all sub-iterations are performed on the fine level.

\section{RESULTS}

The proposed method is applied to a two-dimensional laminar and a two-dimensional turbulent test case. The laminar case is based on [3] and consists of a circular cylinder of diameter $0.1 \mathrm{~m}$ in a channel with height $H=0.41 \mathrm{~m}$, length $L=2.5 \mathrm{~m}$, with an elastic flap behind it of length $l=0.35 \mathrm{~m}$ and thickness $h=0.02 \mathrm{~m}$, see Fig. 1. For the turbulent case a square cylinder with edges of $0.1 \mathrm{~m}$ is used instead of a circular cylinder to increase the shed vorticity. The inflow is a

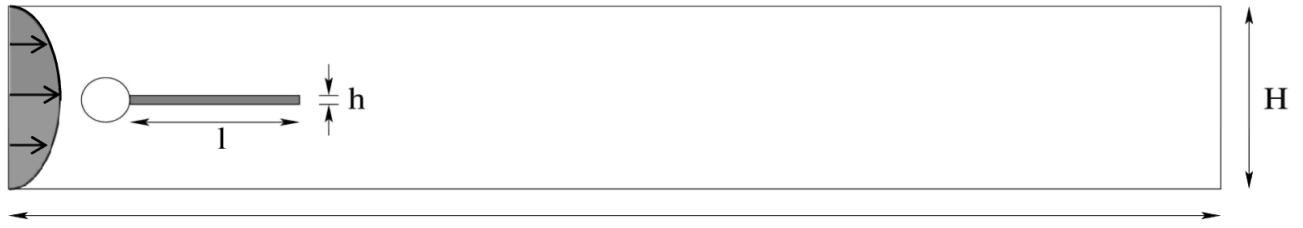

$\mathrm{L}$

FIGURE 1. Two-dimensional testcase.

parabolic velocity profile (see [3] for details) with a mean velocity of 2 and a maximum velocity of $3 \mathrm{~m} / \mathrm{s}$. The solver we use is a compressible Reynolds Averaged Navier-Stokes solver, so compared to the original testcase compressibility is allowed, setting the reference Mach number based on the mean flow velocity to $M_{0}=0.14$. The Reynolds number is based on the mean velocity and cylinder diameter and is $R e=200$ for the laminar case. For the turbulent case we use the Spalart-Allmars turbulence model and $R e=1 \cdot 10^{5}$. The structure is modeled as a linear elastic structure with a density equal to the flow density $\rho=1000 \mathrm{~kg} / \mathrm{m}^{3}$ and a Young's modulus of $E=5.6 \cdot 10^{6} \mathrm{~kg} /\left(\mathrm{m} . \mathrm{s}^{2}\right)$ for the laminar and $E=22.4 \cdot 10^{6} \mathrm{~kg} /\left(\mathrm{m} . \mathrm{s}^{2}\right)$ for the turbulent case. Interpolation between the fluid and structure interfaces is performed using a radial basis function interpolation for transferring structural displacements to the fluid mesh $\left(\mathscr{I}_{f}\right)$ and a nearest neighbor interpolation for transferring the pressure to the structure mesh $\left(\mathscr{I}_{s f}\right)$. Time integration is performed by an implicit, third-order accurate, multi-stage Runge-Kutta scheme with a time step $\Delta t=0.01$ s (for details on interface interpolation and time integration see [4]). Each implicit Runge-Kutta stage is sub-iterated until $\left\|\mathbf{p}_{\Gamma_{s}}^{i}-\hat{\mathbf{p}}_{\Gamma_{s}}^{i}\right\|_{2} \leq 10^{-2}$. 
For the laminar case we simulate in 500 time steps the transient to a periodic state. For the turbulent case the initial condition is taken after the transient to a periodic coupled solution, and only a single period $(\approx 32$ time steps) is simulated. In both computations the flow solver uses 3 grid levels for the multigrid (MG) solver. For the coarse level iterations, we use the intermediate MG mesh. The fine/coarse level meshes consist of 20737 and 5442 cells for the laminar and of 56746 and 15151 cells for the turbulent case. We vary the number of coarse grid sub-iterations before performing a fine grid solve. In Fig. 2 we plot the averaged number of sub-iterations that are required per stage in terms of coarse and fine level iterations. The first datapoint in the plots gives the reference number of sub-iterations
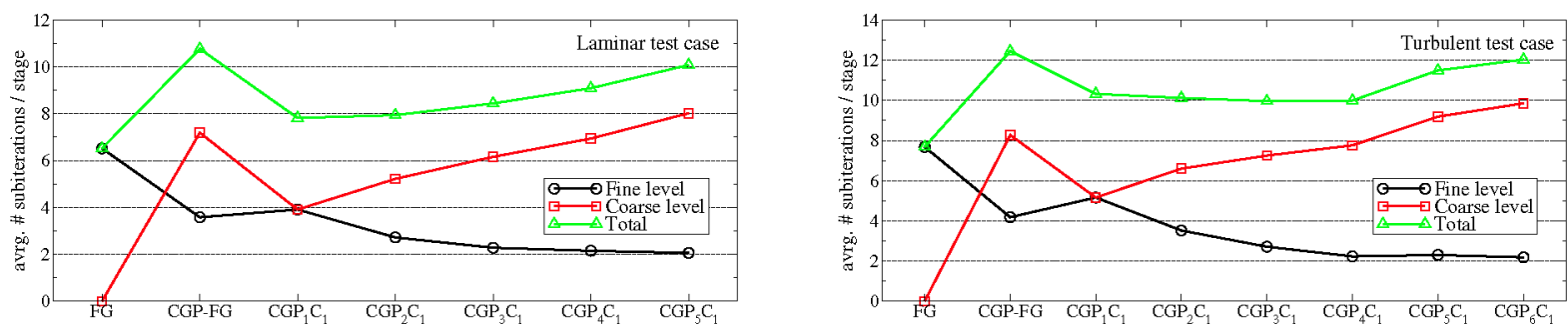

FIGURE 2. Averaged number of coarse and fine grid sub-iterations per stage for the laminar and turbulent case.

as these correspond to Aitken sub-iterations on the fine grid (FG) only. The second datapoint (CGP-FG) separates the sub-iterations on the coarse and fine level. From this computation it was found that the average underrelaxation $\theta$ was almost identical on both levels, indicating that the convergence of the Aitken algorithm is comparable on the fine and coarse grid level. It was, therefore, expected that applying the coarse and fine level sub-iterations alternatingly, should not harm the stability and convergence of the Aitken method. CGP-FG reduces the number of fine grid sub-iterations by roughly $40-50 \%$, but on the other hand increases the total number of sub-iterations more than $60 \%$. Therefore, a lot of computational efficiency gained by performing less fine grid flow solves is lost by the additional coarse level subiterations to perform, which also include moving the fluid mesh, transfer of data between flow and structure and solving the structure. Alternating coarse level/fine level sub-iterations, reduces the number of fine level sub-iterations as well as the total number of sub-iterations compared to CGP-FG. Increasing the number of coarse grid sub-iterations before a fine grid sub-iteration reduces the number of required fine grid solves, which seems to converge to $\approx 2$. Increasing the number of coarse grid sub-iterations even further, only increases the total number of sub-iterations required, without an obvious gain in reduction of fine grid sub-iterations. The most effective schemes are found to be $\mathrm{CGP}_{3} \mathrm{C}_{1}$ for the laminar case and $\mathrm{CGP}_{4} \mathrm{C}_{1}$ for the turbulent case, reducing the number of fine grid iterations by $65-70 \%$ at the expense of an increase in total number of sub-iterations by $25-30 \%$.

\section{CONCLUSIONS}

In this paper we investigated the combination of multi-level acceleration with the Aitken underrelaxation technique applied to a two-dimensional strongly coupled laminar and turbulent test problem. Since the value for the underrelaxation parameter is not significantly different for coarse and fine level sub-iterations, they are used alternatingly, where it is found that performing 3 or 4 coarse level sub-iterations followed by 1 fine level sub-iteration results in the highest gain in efficiency. Although the total number of sub-iterations increases slightly by $25-30 \%$, the number of fine grid iterations was decreased by as much as $65-70 \%$.

\section{REFERENCES}

1. D. Mok, W. Wall, and E. Ramm, "Accelerated iterative substructure schemes for instationary fluid-structure interaction," in First MIT Conference on Computational Fluid and Solid Mechanics, 2001, pp. 1325-1328.

2. A. v. Zuijlen, S. Bosscher, and H. Bijl, Comput. Methods Appl. Mech. Engrg. 196, 1458-1470 (2007).

3. S. Turek, and J. Hron, "Proposal for numerical benchmarking of fluid-structure interaction between an elastic object and laminar incompressible flow," in Fluid-Structure Interaction: Modelling, Simulation, Optimisation, edited by H.-J. Bungartz, and M. Schaefer, Springer, 2006.

4. A. v. Zuijlen, A. d. Boer, and H. Bijl, J. Comput. Phys. 224, 414-430 (2007). 